\title{
Les pertes de substances osseuse mandibulaires : \\ prise en charge prothétique et implantaire
}

\section{The losses of substances osseous mandibular: coverage prothetic and implantaire}

\section{Résumé}

\author{
MOTS-CLEFS : \\ - Implants dentaires, \\ radiothérapie \\ cervico-faciale, perte \\ de substance osseuse \\ mandibulaire
}

\section{KEYWORDS:}

- Dental implant, cervico facial radiotherapy, mandibular defects, mandibular reconstruction

$\operatorname{AOS} n^{\circ} 278-2016$
Les reconstructions des pertes de substance osseuse mandibulaires (suite à un traumatisme ou ò une résection tumorale) doivent être associées ò une chirurgie implantaire.

Cet article montre l'expérience acquise par l'équipe pluridisciplinaire de l'hôpital Cochin ò travers quelques cas cliniques.
Abstract

Mandibular bone reconstruction (after traumatology or tumor surgery) needs to be completed by dental implant placement. This case report illustrates the satisfactory functional and aesthetic results obtained by the multidisciplinary team of Cochin Hospital.

\begin{tabular}{|l|}
\hline Leva DJAVANMARDI' \\
GuY PRINC' \\
Gilles GREUX' \\
Michel KURC' \\
'Service de Stomatologie et Chirurgie maxillo-faciale, Groupe hospitalier Cochin \\
Saint-Vincent de Paul, 27, rue du Faubourg Saint-jacques, 75679 Paris Cedex 14. \\
\hline
\end{tabular}

\section{INTRODUCTION}

Le présent travail montre l'expérience acquise dans le domaine des reconstructions prothétiques implanto-portées mandibulaires. Les pertes de substance osseuse peuvent résulter de traumatismes ou de résections tumorales plus ou moins élargies.

Les cas cliniques présentés sont pris en charge à l'hôpital Cochin dans le cadre du Diplôme d'Université de chirurgie et prothèses implantologiques.
CAS CLINIQUE $N^{\circ} 1$ : CARCINOME ÉPIDERMOÏDE PELVI-MANDIBULAIRE T1, No

Patiente âgée de 60 ans, édentée totale ; découverte d'une lésion ulcéro-indurée du plancher antérieur. La biopsie conclut à un carcinome épidermoïde. Après le bilan, la tumeur est classée T1N0. Le protocole thérapeutique est chirurgical. 


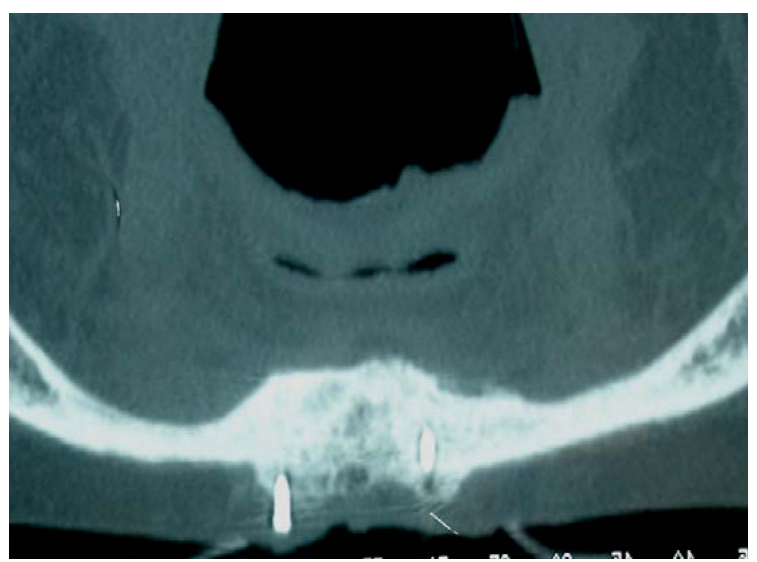

$\triangle$ Fig. 1.

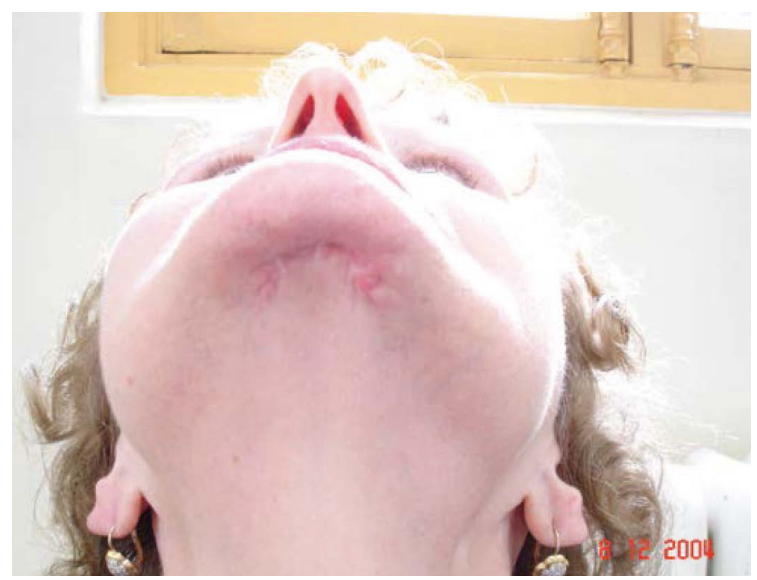

$\triangle$ Fig. 2.

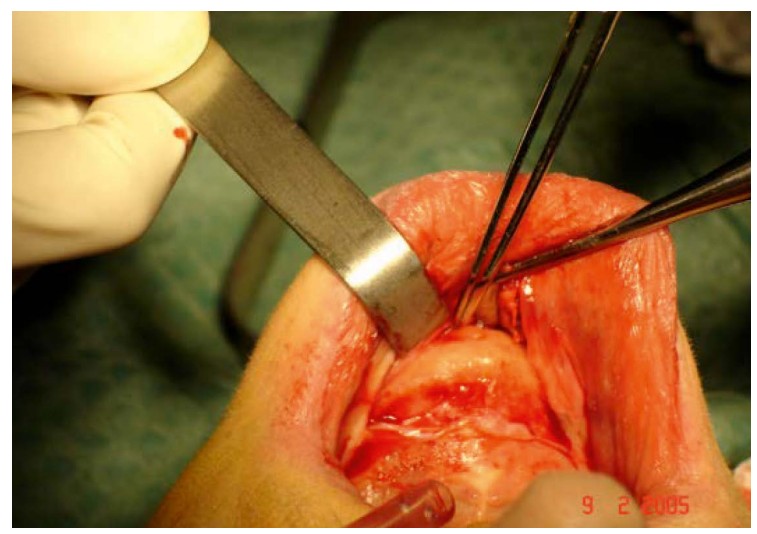

$\triangle$ Fig. 4.

CAS CLINIQUE $N^{\circ} 2$ : CARCINOME ÉPIDERMOÏDE PELVI-MANDIBULAIRE ANTÉROLATÉRAL GAUCHE T3, N2

Un patient âgé de 45 ans a été opéré il y a une quinzaine d'années, suite au diagnostic de carcinome épidermoïde pelvi-mandibulaire antéro-latéral gauche classé T3, N2.

Le traitement était uniquement chirurgical : résection mandibulaire interruptrice associée à un curage
Dans un deuxième temps, une greffe osseuse est pratiquée pour augmenter la dimension verticale symphysaire (fig. 1).

I La voie d'abord utilisée est sousmentonnière, permettant un abord de la symphyse mandibulaire en respectant le vestibule inférieur (fig. 2, 3).

I La greffe osseuse (prélèvement iliaque) est ostéosynthésée au niveau du bord basilaire par des vis.

16 mois plus tard, (après ablation des vis d'ostéosynthèse) par voie endobuccale, mise en place des deux implants symphysaires (fig. 4, 5).

$\checkmark$ Ainsi deux boutons pressions permettent la stabilisation de la prothèse totale mandibulaire.

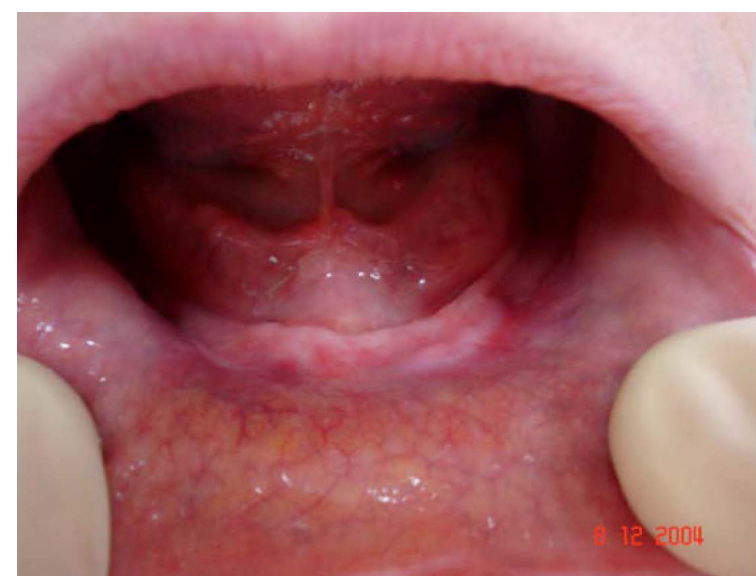

$\triangle$ Fig. 3.

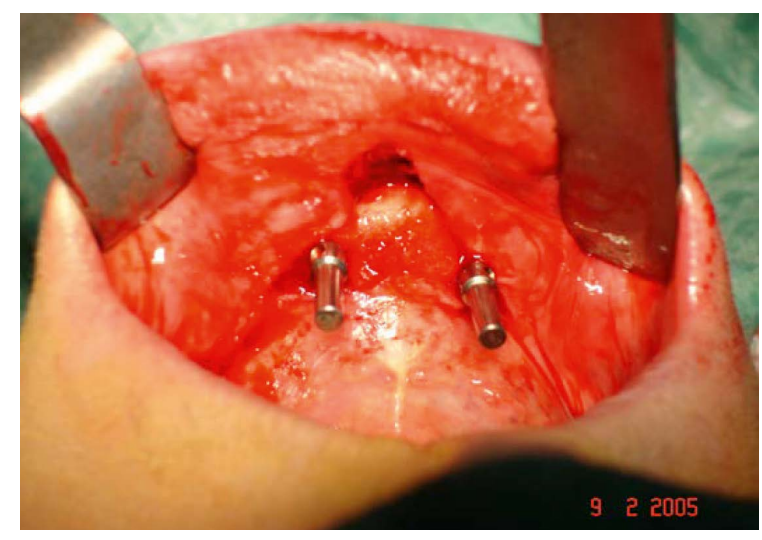

$\triangle$ Fig. 5.

ganglionnaire cervical et reconstruction mandibulaire du secteur antéro-latéral par un greffon costal ostéosynthésé par des fils d'acier (fig. 6).

L'état locorégional est satisfaisant. La dent 47 est à extraire. La prothèse dentaire est instable, rendant impossible la fonction masticatoire.

Le volume osseux disponible évalué par un examen tomodensitométrique n'étant pas suffisant pour permettre une réhabilitation implantaire, il est décidé de réaliser un prélèvement osseux crânien (fig. 7).

Celui-ci est adossé à la reconstruction mandibulaire et synthésé par des vis (fig. 8, 9). 

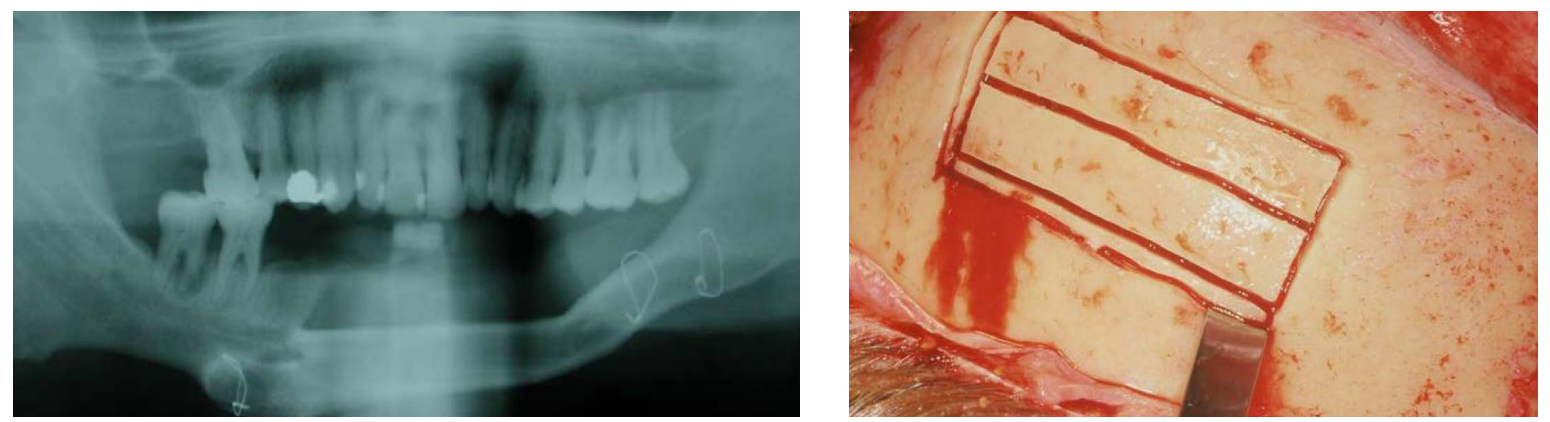

$\triangle$ Fig. 6.

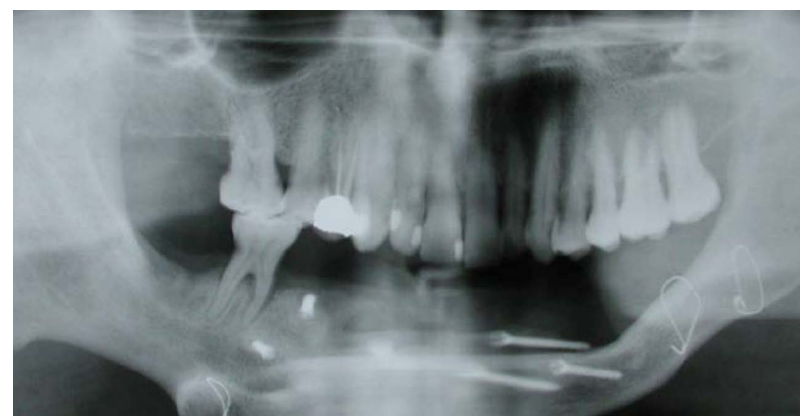

$\triangle$ Fig. 8.

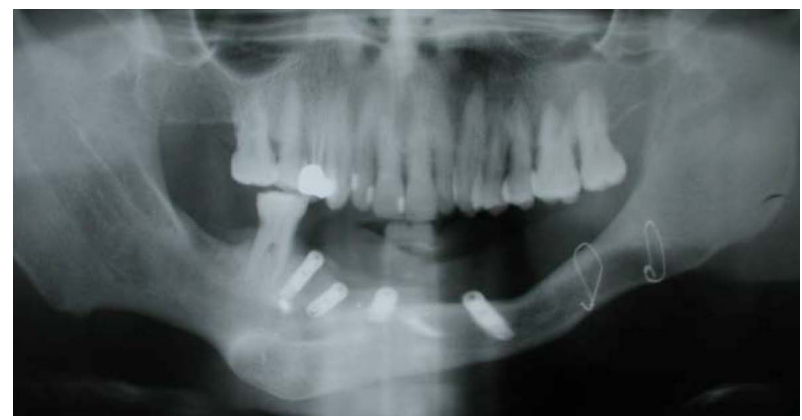

$\triangle$ Fig. 10.

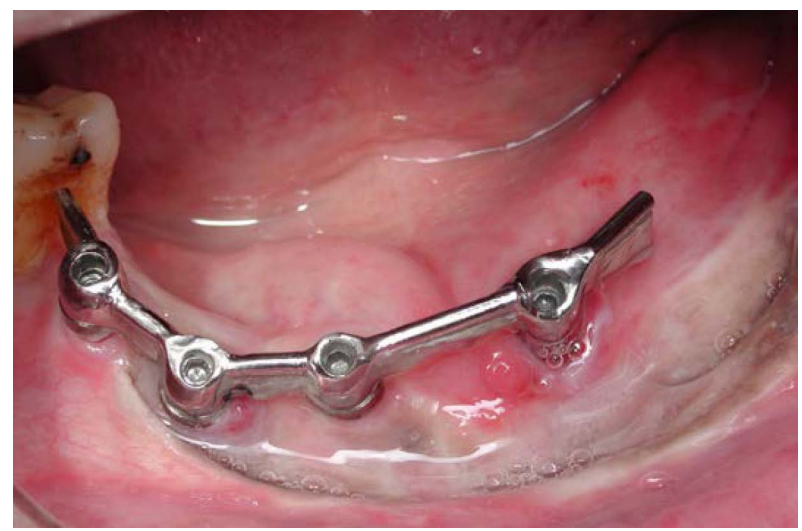

$\triangle$ Fig. 7.

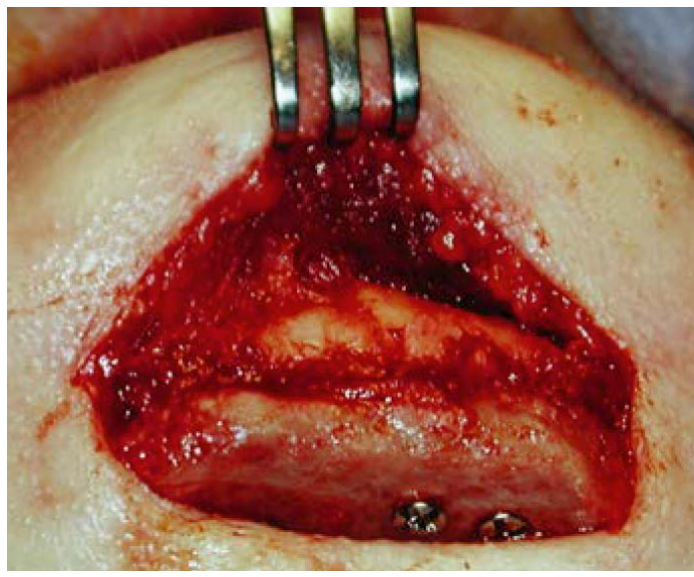

Fig. 9.

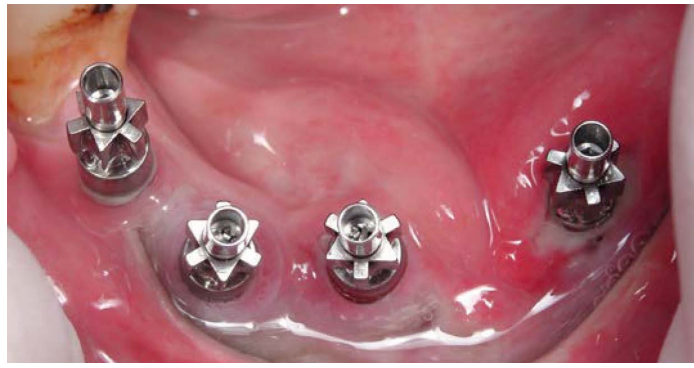

$\triangle$ Fig. 11.

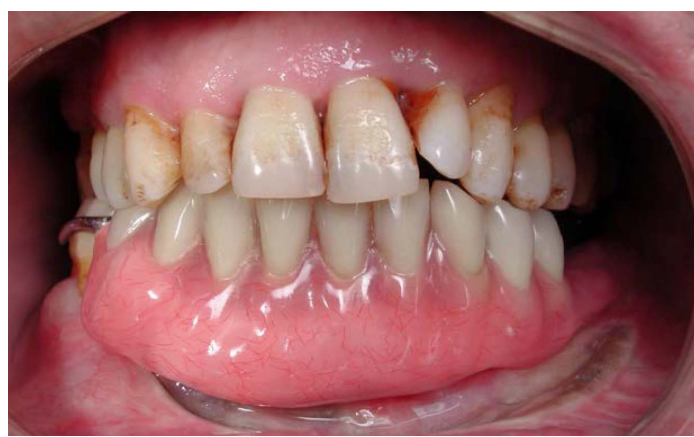

$\triangle$ Fig. 12.

ISix mois plus tard après réévaluation du volume osseux disponible, 4 implants mandibulaires (fig. 10) sont positionnés (uniquement en fonction des considérations anatomiques).

Après approfondissement du vestibule par une greffe cutanée, mise en place des bagues de cicatrisation et réalisation de la barre implantoportée (fig. 11, 12). Réalisation de la prothèse dentaire (fig. 13, 14).

$\triangle$ Fig. 13.

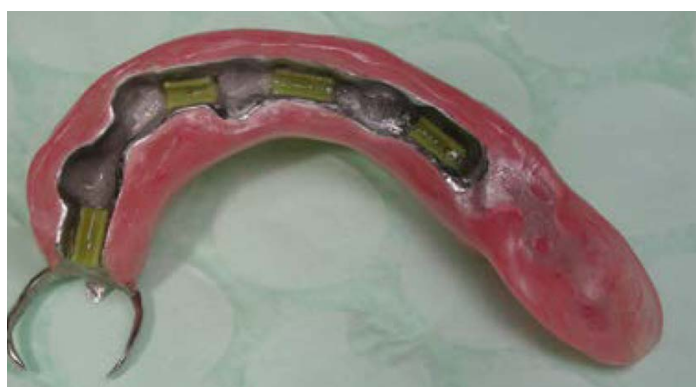

$\triangle$ Fig. 14. 


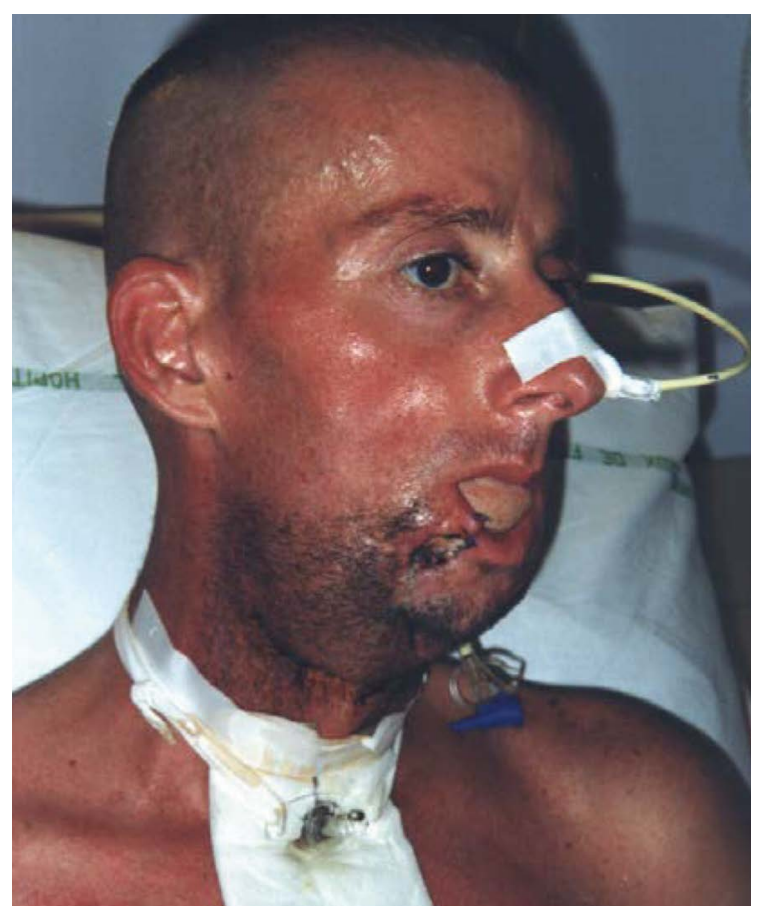

$\triangle$ Fig. 15.

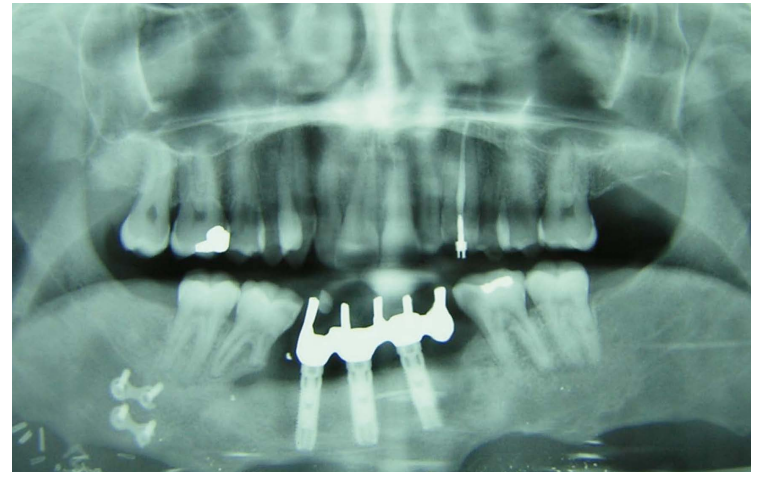

Fig. 16.

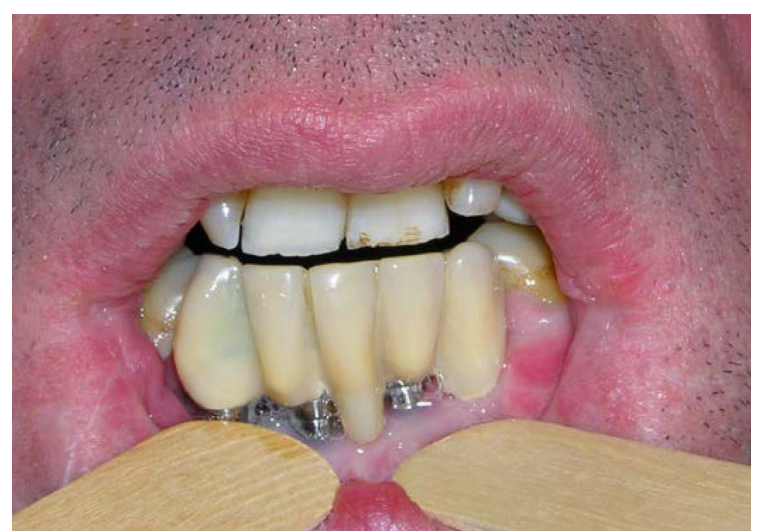

$\triangle$ Fig. 17.
CAS CLINIQUE $N^{\circ} 3$ :

PERTE DE SUBSTANCE OSSEUSE COMPOSITE DU TIERS INFÉRIEUR

DE LA FACE RÉSULTANT

D'UN TRAUMASTISME BALISTIQUE

Un patient âgé d'une trentaine d'années, victime d'un traumatisme balistique (porte d'entrée sous-mentonnière avec perte de substance de l'étage inférieur de la face) est admis en urgence : trachéotomie et mise en place d'une sonde gastrique.

Secondairement le patient bénéficie d'une reconstruction de la région antérieure mandibulaire par un transfert osseux de fibula (fig. 15).

Le résultat morphologique et fonctionnel est jugé incomplet du fait de l'importante rétrusion de l'étage inférieur de la face, avec obstruction de l'oropharynx par la langue.

Une distraction osseuse mandibulaire bilatérale permet de supprimer la sonde gastrique et la trachéotomie. Secondairement, on décide la mise en place de 3 implants dentaires dans la région édentée symphysaire (sur le transfert osseux) et réalisation de la prothèse (fig. 17, 18).

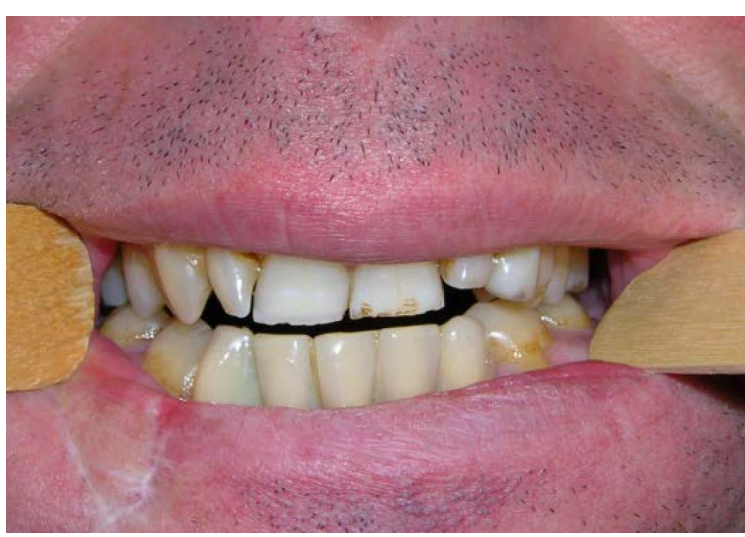

$\triangle$ Fig. 18. 
CAS CLINIQUE N ${ }^{\circ} 4:$ CARCINOME ÉPIDERMOÏDE PELVI-MANDIBULAIRE ANTÉRIEUR $\mathrm{T}_{3}, \mathrm{~N}_{3}$

Chez une patiente âgée de 45 ans, alcoolo-tabagique, découverte tardive d'un carcinome épidermoïde pelvimandibulaire antérieur T3, N3. Le protocole thérapeutique associe une chirurgie d'exérèse tumorale ganglionnaire, cervicale et une reconstruction immédiate de la région antérieure par une attelle, recouverte par un lambeau myocutané. La chirurgie est suivie d'une radiothérapie cervicofaciale.

Dans un deuxième temps (fig. 19) dépose de l'attelle mandibulaire et transfert micro-anastomosé (fibula) pour la reconstruction antérieure (les brides cervicales résultent de la radiothérapie post-opératoire).

Sur le panoramique (fig. 20) : plaque d'ostéosynthèse et 3 implants.

Mise en place des 3 boutons pression (fig. 21) et de la prothèse dentaire (fig. 22).

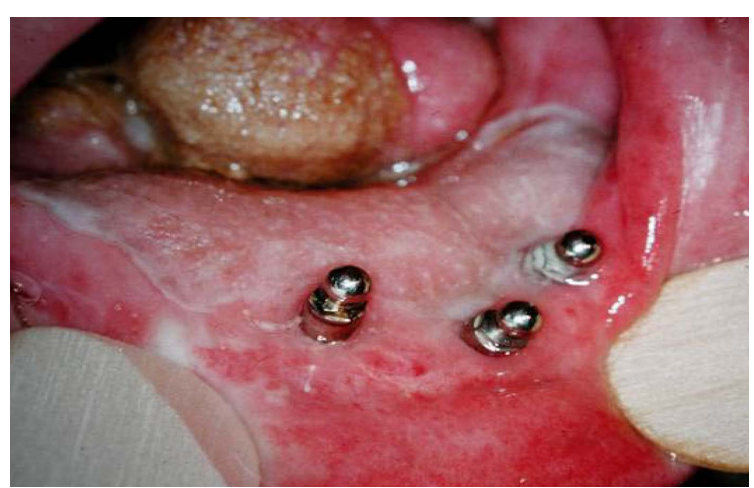

$\triangle$ Fig. 21.

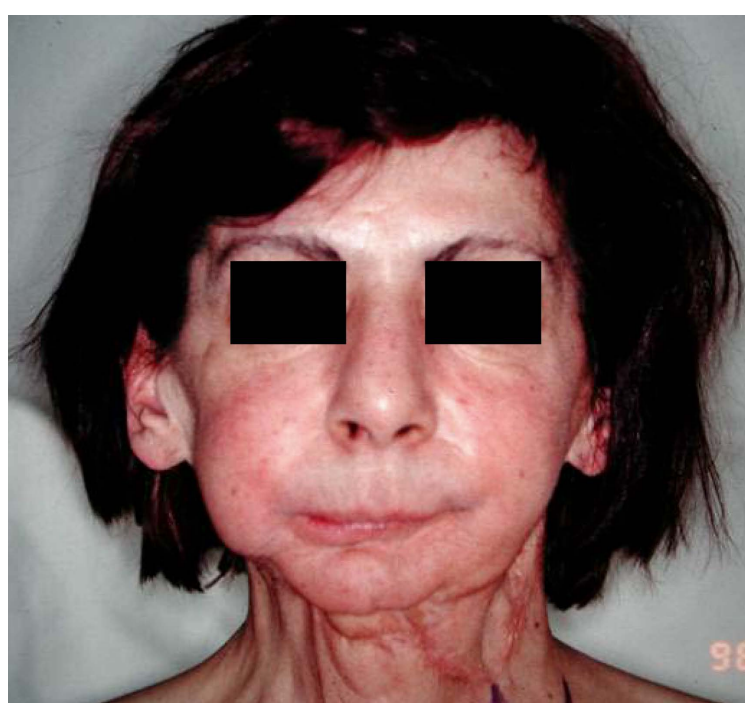

Fig. 19.

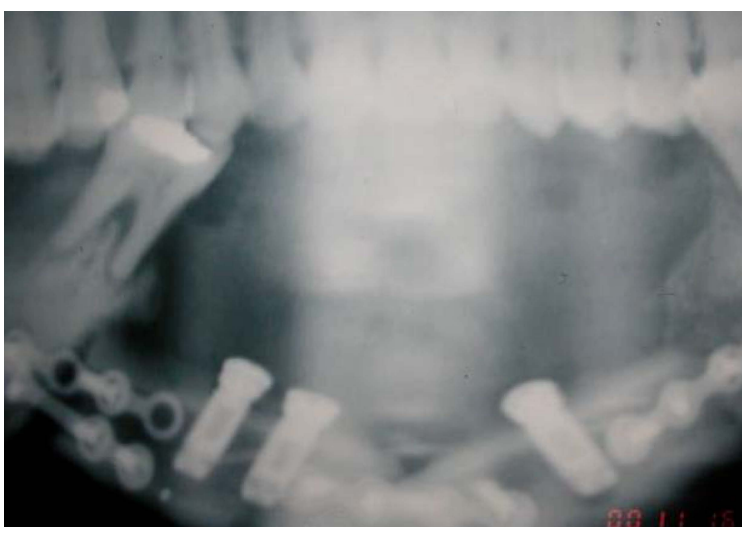

$\triangle$ Fig. 20.

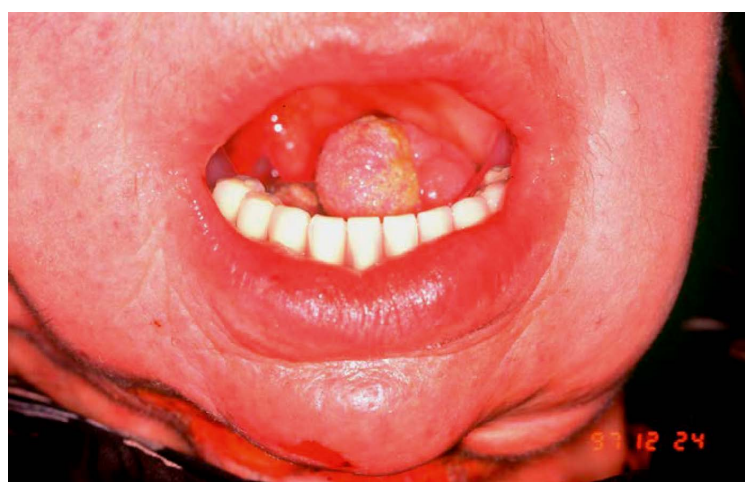

$\triangle$ Fig. 22. 


\section{CAS CLINIQUE $\mathrm{N}^{\circ} 5$ : \\ CARCINOME ÉPIDERMOÏDE DE LA COMMISSURE INTERMAXILLAIRE T3, N3}

Patient âgé de 51 ans, alcoolo-tabagique présentant une lésion ulcéroindurée de la commissure inter-maxillaire gauche. La biopsie conclut à un carcinome épidermoïde. Le bilan au niveau des VADS est normal. Le protocole thérapeutique associe :

$\checkmark$ une résection tumorale (mandibulaire et maxillaire), un curage ganglionnaire cervical radical et une radiothérapie cervico-faciale postopératoire ;

$\checkmark$ une prothèse obturatrice maxillaire est réalisée en post-opératoire immédiat. Le patient étant édenté total, la prothèse dentaire inférieure est instable ; on décide de positionner 2 ans plus tard, 3 implants mandibulaires (fig. 23).

Prothèse obturatrice maxillaire (fig. 26).

La prothèse dentaire est stabilisée sur la barre implanto-portée ; elle permet au patient une fonction masticatoire correcte (fig. 25-27).

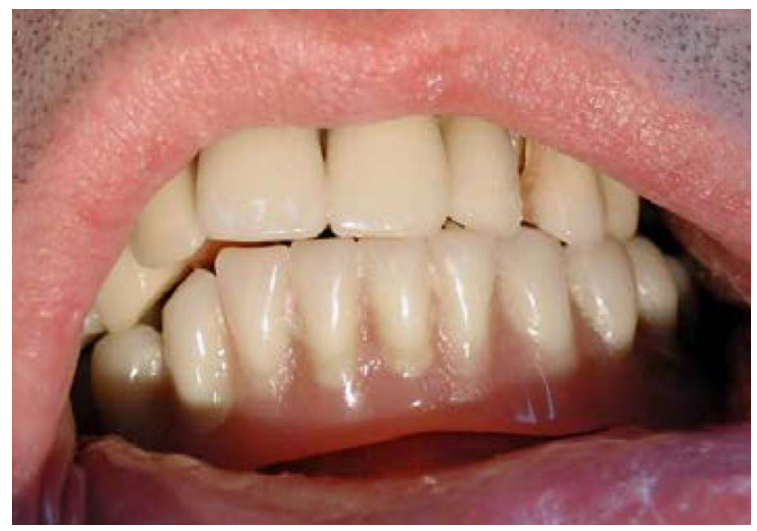

$\triangle$ Fig. 25.

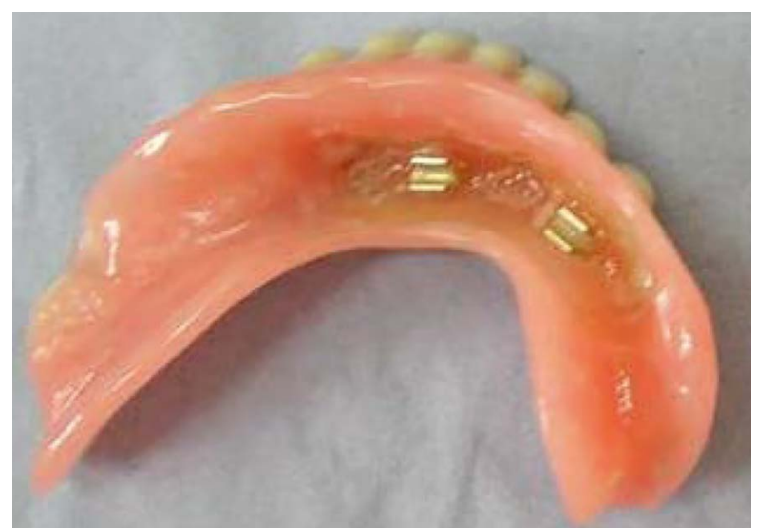

$\triangle$ Fig. 27.

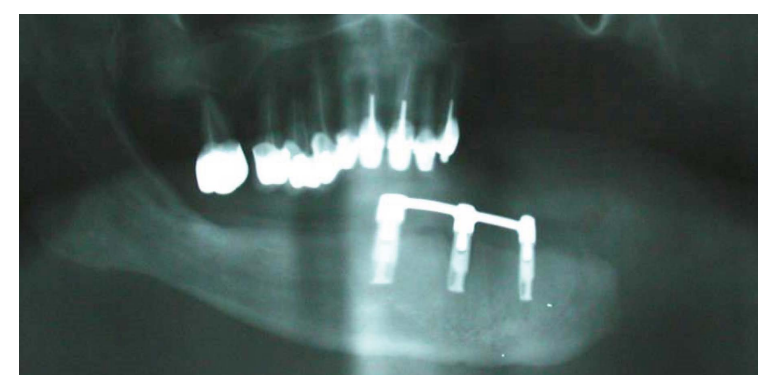

$\triangle$ Fig. 23.

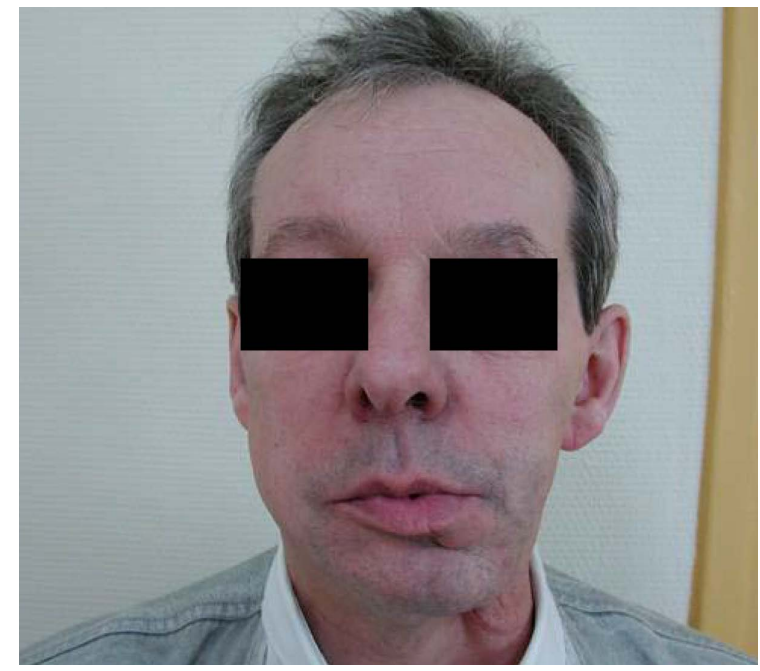

$\triangle$ Fig. 24.

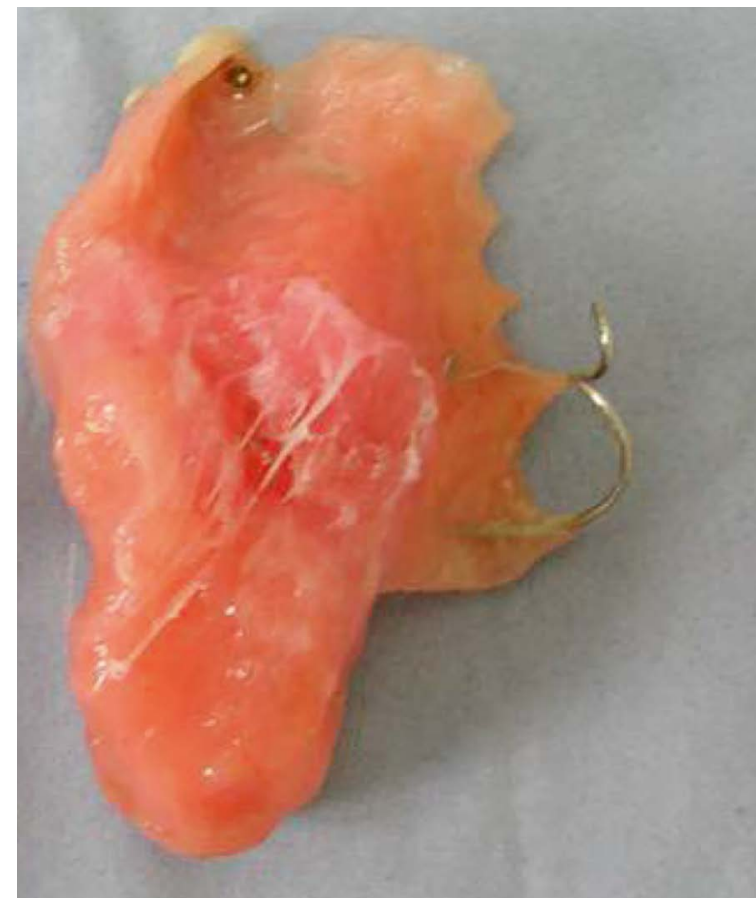

$\triangle$ Fig. 26. 
CAS CLINIQUE N 6 : AMÉLOBLASTOME MANDIBULAIRE

Une résection mandibulaire interruptrice antérolatérale a été pratiquée il y a une dizaine d'année pour un amélobastome avec une reconstruction immédiate par une greffe osseuse (iliaque).

La mise en place de 4 implants (fig. 31 a) est indiquée pour stabiliser la prothèse dentaire.

La photographie 29 montre l'importance de la hauteur libre prothétique.

Après une augmentation du volume osseux par une greffe d'apposition; mise en place de quatre implants dentaires (fig. $31 \mathrm{~b}$ ).

Réalisation de la prothèse dentaire (fig. 32, 33, 34, 35, $36,37,38,39)$.

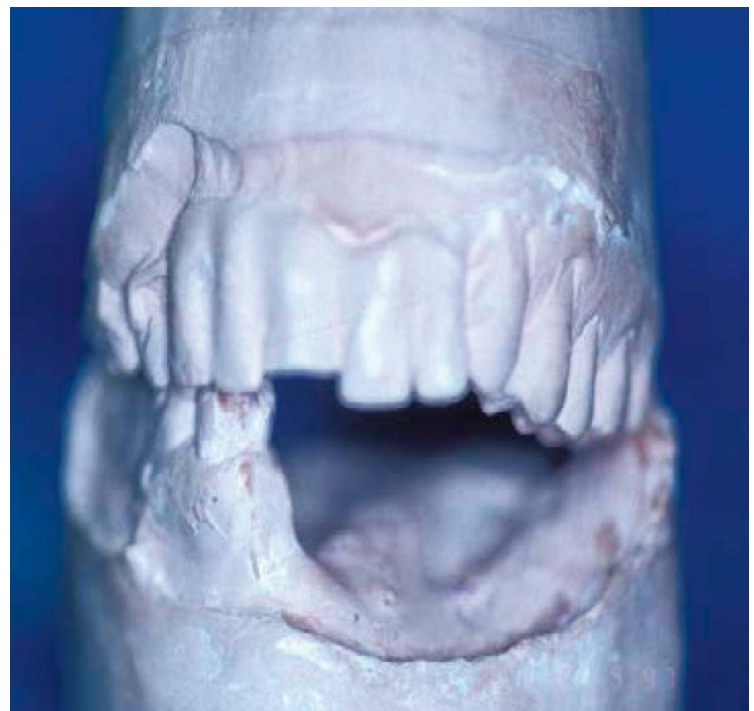

$\triangle$ Fig. 29.

Fig. 31 .

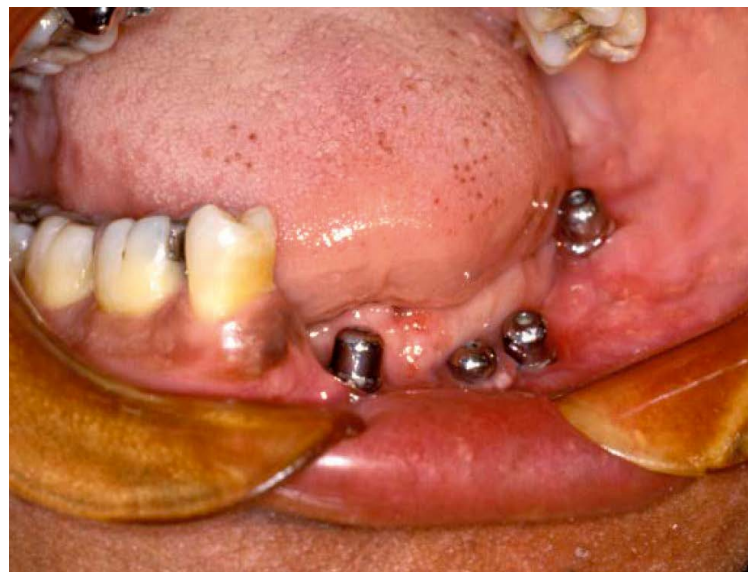

$\triangle$ Fig. 32.

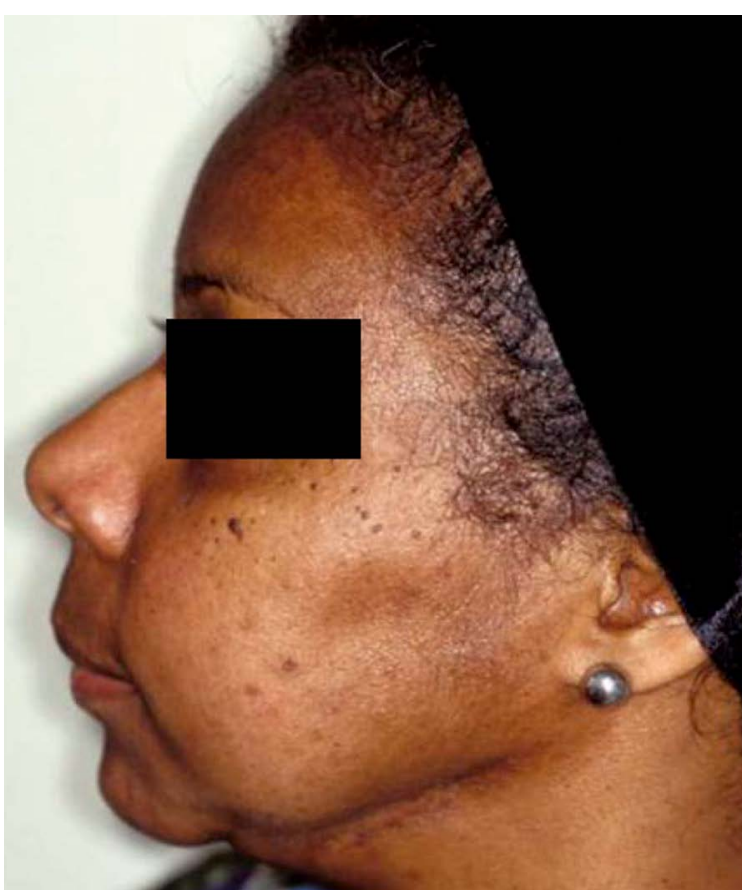

$\triangle$ Fig. 28.

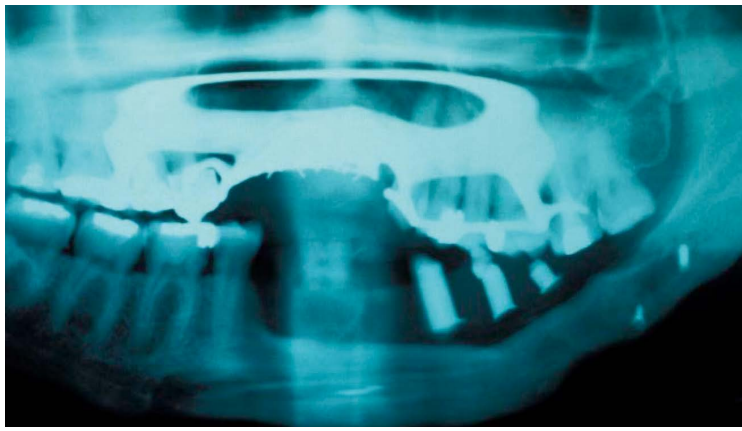

Fig. 30.
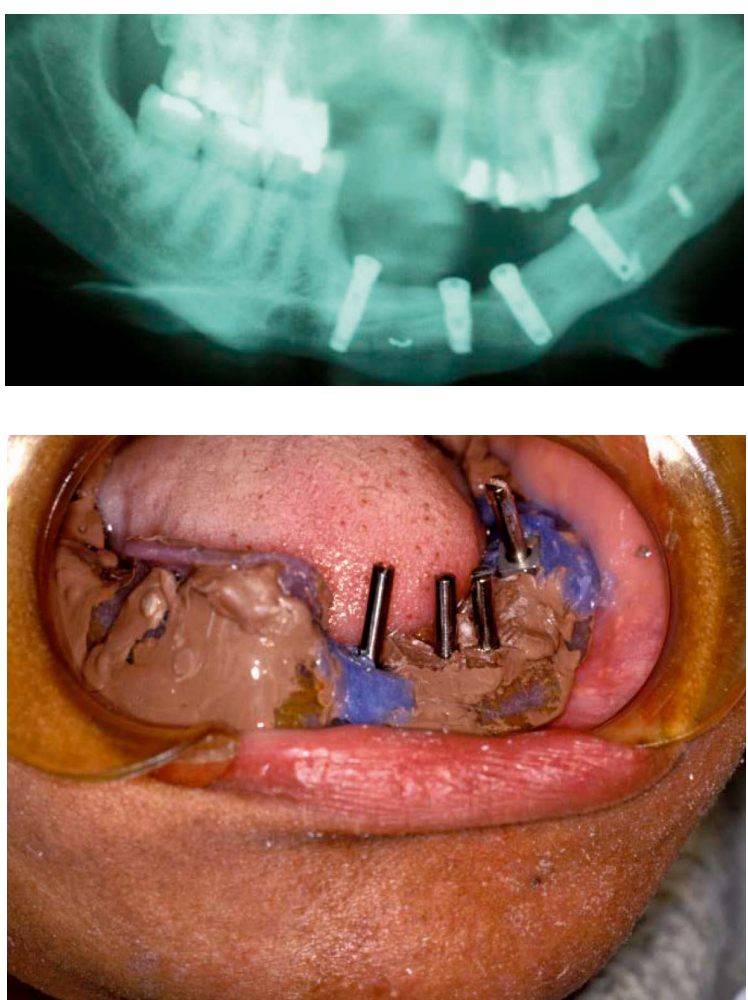

$\triangle$ Fig. 33. 
Cas clinique Djavanmardi L., Princ G., Greux G., Kurc M.

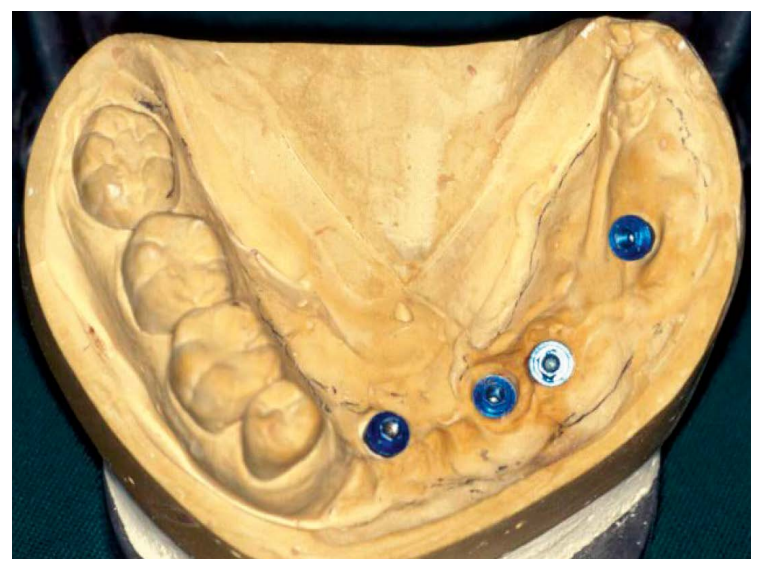

$\triangle$ Fig. 34.

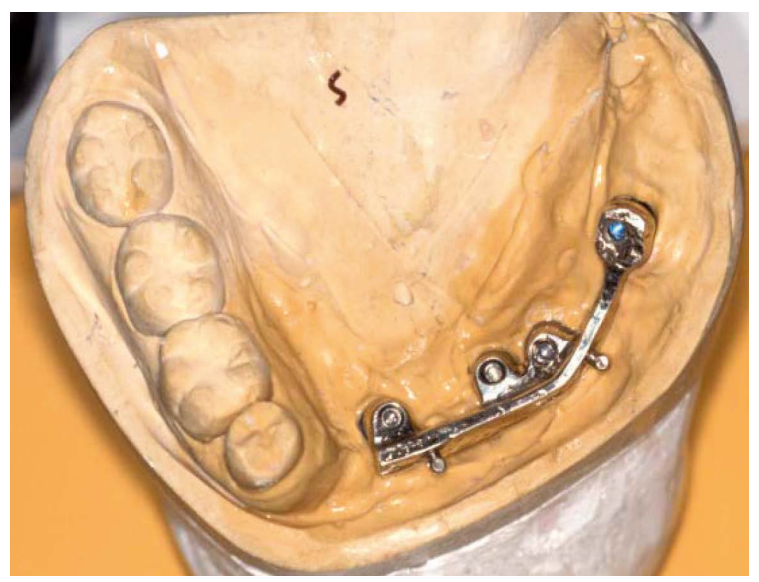

$\triangle$ Fig. 36.

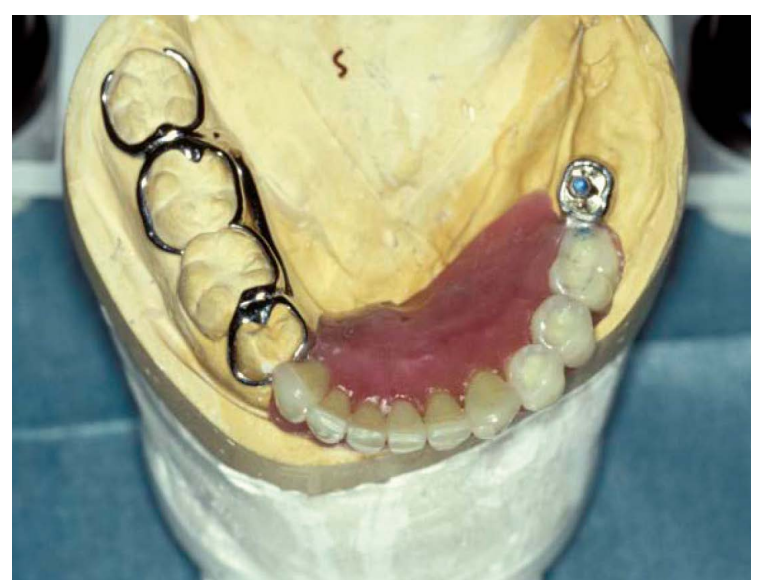

Aig. 38.

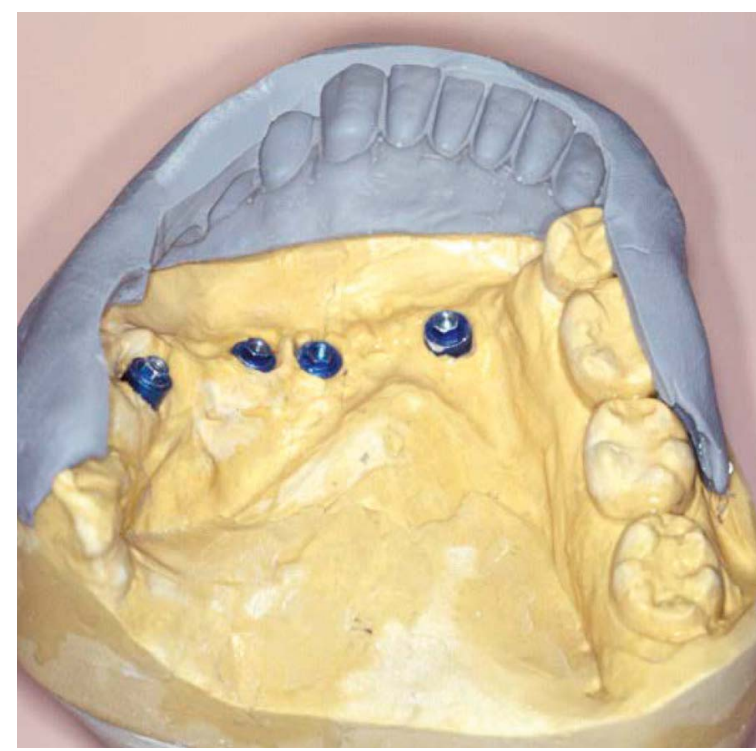

Fig. 35.

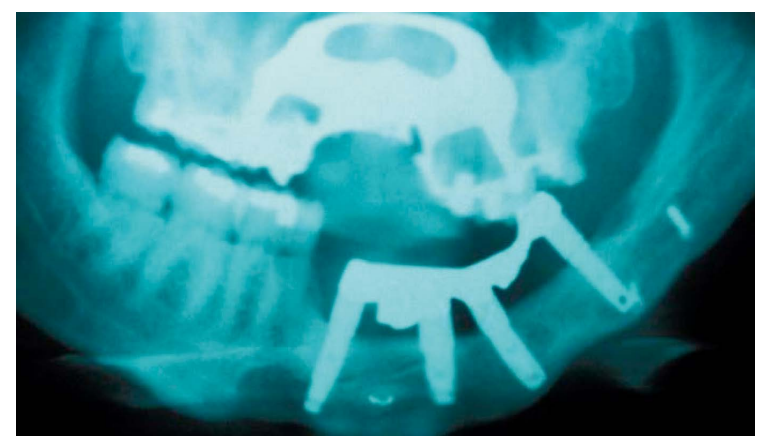

$\triangle$ Fig. 37.

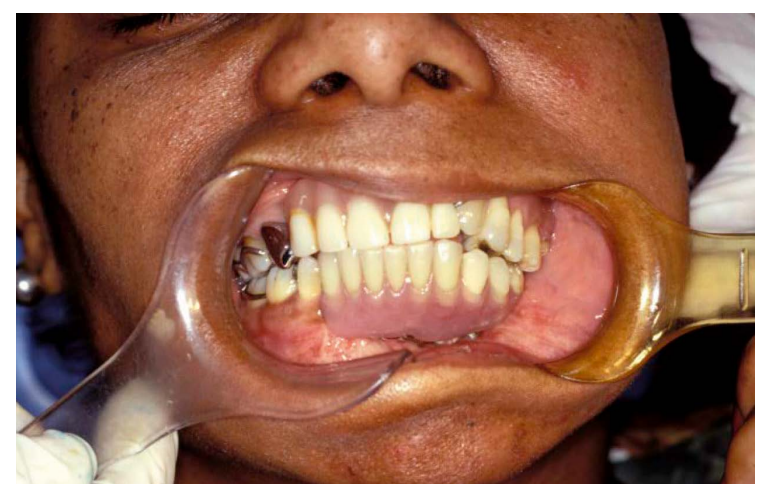

$\triangle$ Fig. 39. 


\section{CAS CLINIQUE $N^{\circ} 7$ : AMÉLOBLASTOME MANDIBULAIRE}

Il s'agit d'un autre cas d'améloblastome mandibulaire ayant nécessité la dépose du bridge 3435 36 37, l'avulsion de la dent incluse ectopique 38 et l'énucléation curetage de la lésion mandibulaire (fig. 40, 41, 42).

9 mois plus tard, le panoramique de contrôle montre une ossification complète de la perte de substance osseuse mandibulaire (fig. 43).

Mise en place de 3 implants mandibulaires et réalisation de la prothèse implanto-portée (fig. 44).

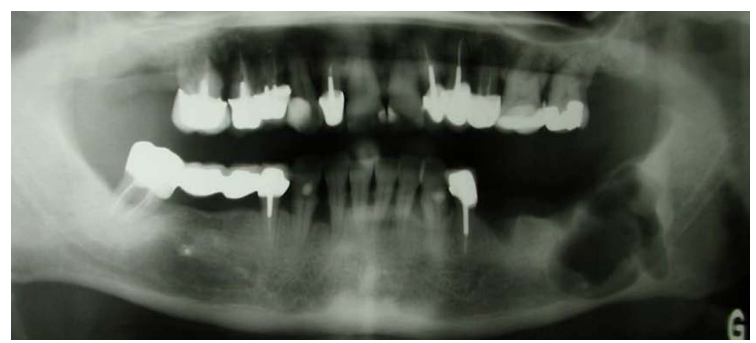

$\triangle$ Fig. 42.

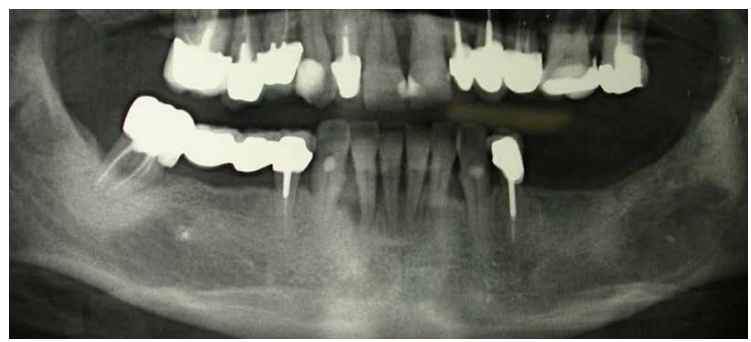

Fig. 43.

\section{CONCLUSION}

Les quelques cas cliniques présentés illustrent les progrès de la réhabilitation prothétique grâce aux implants dentaires.

Une telle thérapeutique rend un service inestimable pour nos patients, modifiant leur qualité de vie, d'un

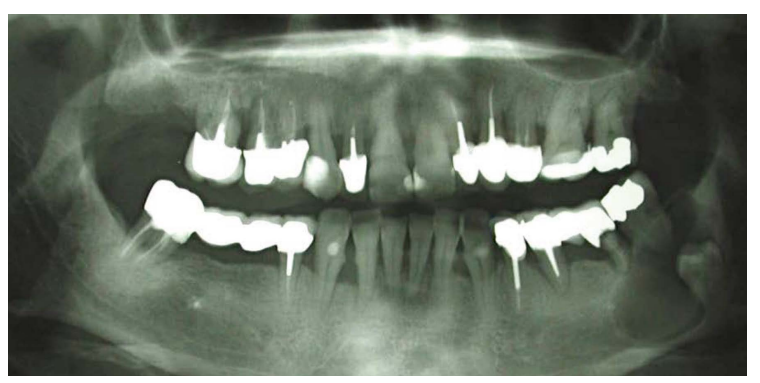

$\triangle$ Fig. 40.

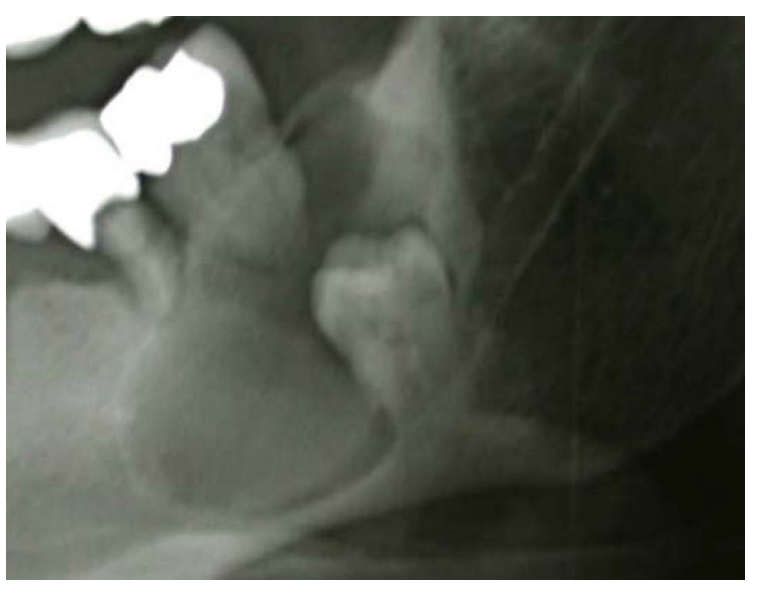

$\triangle$ Fig. 41.

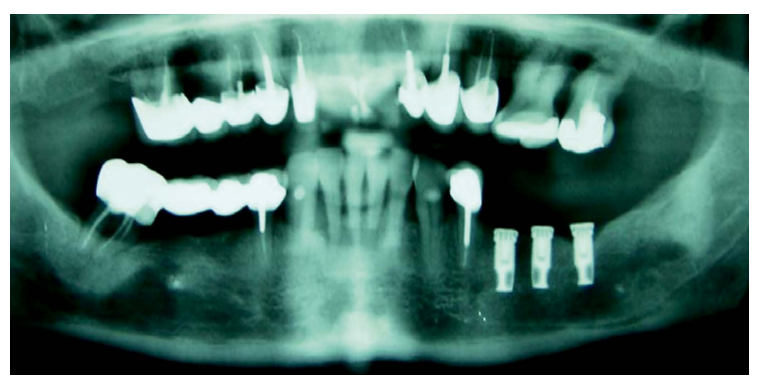

Fig. 44.

point de vue fonctionnel (masticatoire), esthétique et surtout psychologique.

La radiothérapie cervico-faciale ne constitue pas un critère d'exclusion.

En fait, le problème essentiel réside dans l'absence de prise en charge financière des traitements implantaires. 